\title{
AlEJAndro MELlE-HERnÁndeZ On polar invariants of hypersurface singularities
}

Annales de la faculté des sciences de Toulouse $\sigma^{e}$ série, tome $9, \mathrm{n}^{\circ} 4$ (2000), p. 671-688

<http://www.numdam.org/item?id=AFST_2000_6_9_4_671_0>

(C) Université Paul Sabatier, 2000, tous droits réservés.

L'accès aux archives de la revue «Annales de la faculté des sciences de Toulouse » (http://picard.ups-tlse.fr/ annales/) implique l'accord avec les conditions générales d'utilisation (http://www.numdam.org/conditions). Toute utilisation commerciale ou impression systématique est constitutive d'une infraction pénale. Toute copie ou impression de ce fichier doit contenir la présente mention de copyright.

\section{NumDam}

Article numérisé dans le cadre du programme Numérisation de documents anciens mathématiques http://www.numdam.org/ 


\title{
On polar invariants of hypersurface singularities ${ }^{(*)}$
}

\author{
Alejandro Melle-HernándeZ ${ }^{(1)}$
}

RÉSUMÉ. - Dans ce travail on donne une borne pour l'ordre de détermination topologique de certains germes de fonctions holomorphes. Soit $f:\left(\mathbb{C}^{n+1}, 0\right) \rightarrow(\mathbb{C}, 0)$ un germe de fonction holomorphe tel que son lieu des zéros $(V, 0)$ soit une hypersurface à singularité isolée dont le cône tangent définit une hypersurface projective de $\mathbb{P}^{n}$ ayant seulement des singularités isolées. En s'inspirant du travail de B. Teissier la courbe polaire, les invariants polaires et les quotients polaires de $(V, 0)$ sont étudiés. De plus, les résultants obtenus sont appliqués pour caractériser une partie de la strate á $\mu$-constant de la déformation miniverselle de $(V, 0)$.

Abstract. - In the present work an upper bound for the topological determinacy order of some germs of holomorphic functions is given. Let $f:\left(\mathbb{C}^{n+1}, 0\right) \rightarrow(\mathbb{C}, 0)$ be a germ of holomorphic function such that its zero locus $(V, 0)$ is an isolated singularity whose projectivized tangent cone at the origin has only isolated singularities. Following B. Teissier's work, the polar curve, polar invariants and polar quotients of $(V, 0)$ are studied. Moreover our results are applied for studying the equisingular stratum of the germ $(V, 0)$.

(*) Reçu le 23 février 1998, accepté le 19 décembre 2000

(1) Departamento de Álgebra. Faculdad de CC Matemáticas, Universidad Complutense de Madrid, Madrid 28040. España

e-mail: amelle@eucmos.sim.ucm.es

This work was supported in part by PB94-0291 and by an Acción Integrada HispanoFrancesa 950144. 


\section{Introduction}

In this paper we deal with germs $f:\left(\mathbb{C}^{n+1}, 0\right) \rightarrow(\mathbb{C}, 0)$ of holomorphic functions and with their topological classification. Recall that two germs $f$ and $g$ are topologically equivalent, or $C^{0}$-equivalent, if and only if there exists a local homeomorphism $\varphi: \mathbb{C}^{n+1} \rightarrow \mathbb{C}^{n+1}$ with $\varphi(0)=0$ such that $f \circ \varphi=g$. The germ $f$ is $r$ - $C^{0}$-determined if any germ $g$ with $f-g \in \mathfrak{m}^{\mathfrak{r}+1}$ is $C^{0}$-equivalent to $f,(\mathfrak{m}$ is the maximal ideal of $\mathbb{C}\{\bar{z}\})$. If some $r$ exists then $f$ is finitely determined and the minimum of such $r$ is called the topological determinacy order of $f$ which is denoted by several authors by $\operatorname{Suf}(\mathrm{f})$.

It is known, see [4] and its references, that the Lojasiewicz exponent $\mathrm{E}(f)$ of $f$ is related with the topological determinacy order of $f$ as follows: $\operatorname{Suf}(\mathrm{f})=[\mathrm{E}(f)]+1$ where $[x]$ denotes the integer part of $x$.

In order to find the integer $\operatorname{Suf}(\mathrm{f}), \mathrm{B}$. Teissier related it with some invariants of the zero locus $(V, 0)$ of $f$ whenever $(V, 0)$ has an isolated hypersurface singularity, [21]. B. Teissier introduced the notion of polar curve $\Gamma_{H}$ associated to $f$ and a generic hyperplane $H \subset\left(\mathbb{C}^{n+1}, 0\right)$. The irreducible components (branches) of $\Gamma_{H}$ are very closely related to topological properties of the germ $(V, 0)$. Let $\gamma_{q}$ be a branch of $\Gamma_{H}$. This branch $\gamma_{q}$ has associated two positive integers: the polar invariants $\left(e_{q}, m_{q}\right)$. If we set the polar quotients $\left\{\frac{e_{q}}{m_{q}}\right\}$ then B. Teissier proved that $\mathrm{L}(f)$ is the supremum of polar quotients $\frac{e_{q}}{m_{q}},[21]$ Corollaire 2 .

From this starting point, the polar curve of a plane curve singularity has been studied. Let $(C, 0) \subset\left(\mathbb{C}^{2}, 0\right)$ be the germ of an isolated plane curve singularity. If $(C, 0)$ is irreducible then M. Merle, [19], described the sequence of polar quotients in terms of the Puiseux exponents of $(C, 0)$. After that several authors have given similar descriptions for the polar curve and its related invariants: F. Delgado [5], Lê D.T., F. Michel and C.Weber [12], E. Casas [2] [3], E. García-Barroso [6].

As far as the author knows, in higher dimensions there are not such results. Let $f=f_{d}+f_{d+k}+\ldots$ (higher degree terms) be the Taylor expansion of $f$ around 0 . Thus the zero locus of the homogeneous polynomial $f_{d}$ is the tangent cone of $(V, 0)$ at the origin. Let $D$ denote the projectivized tangent cone of $(V, 0)$ at the origin, i.e. the projective hypersurface $D \subset \mathbb{P}^{n}$ defined by the zero locus of $f_{d}$.

B. Teissier, see for instance Proposition 11 in [23], showed that $(V, 0) \subset$ $\left(\mathbb{C}^{n+1}, 0\right)$ is topologically equivalent to the singularity $\left(\left\{f_{d}=0\right\}, 0\right) \subset$ $\left(\mathbb{C}^{n+1}, 0\right)$ if and only if, for every $i \in\{1, \ldots, n+1\}$, the Milnor number $\mu^{(i)}(V, 0)$ of the general plane section of dimension $i$ of $(V, 0)$ is $(d-1)^{i}$. This 
conditions imply that the singularity $(V, 0)$ can be resolved by blowing-up once the origin.

In this paper we generalize the result of $\mathrm{B}$. Teissier for some hypersurfaces. Indeed we make a partial description of the polar curve for any hypersurface singularity $(V, 0)$ which verifies the following condition:

$(*)$ The germ $(V, 0)$ is an isolated hypersurface singularity and the tangent cone of $(V, 0)$ at the origin defines a projective hypersurface $D \subset \mathbb{P}^{n}$ with at most isolated singularities.

For instance every isolated plane curve singularity or any isolated surface singularity whose projectivized tangent cone $D$ is a reduced curve in $\mathbb{P}^{2}$ verify condition $(*)$.

One of the results of this work is to give a decomposition of the polar curve for hypersurfaces $(V, 0)$ satisfying condition $(*)$. Using this decomposition of the polar curve we will give a bound for $\operatorname{Suf}(f)$.

A first bound for $\operatorname{Suf}(\mathrm{f})$ is the Milnor number of the singularity $(V, 0)$. The Milnor number $\mu(V, 0)$ is computable from $f$ but it is not a sharp bound. Our goal is to obtain a better bound of $\operatorname{Suf}(\mathrm{f})$ than $\mu(V, 0)$ without computing the branches of the polar curve $\Gamma_{H}$.

Suppose that $(V, 0)$ verifies condition $(*)$, then the strict transform $\tilde{V}$ of $V$ after blowing-up once the origin has at most isolated singularities. For every singular point $\widetilde{P}$ of $\widetilde{V}$ there is a well-defined point of $\operatorname{Sing}(D)$ (denoted by $P$ ). It means that the set of singular points of $\widetilde{V}$ determines a subset of $\operatorname{Sing}(D)$. Let $\mathcal{Z}\left(f_{d+k}\right) \subset \mathbb{P}^{n}$ be the divisor defined by $f_{d+k}$. The main contribution of the present paper is related to the evaluation of the contribution of the singular points of the strict transform $\widetilde{V}$ in the behavior of the polar quotients. Let $\mathcal{A}$ be the set of points $\widetilde{P}$ of $\widetilde{V}$ such that $\widetilde{P} \in \operatorname{Sing}(\widetilde{V})$ and the associated point $P \in \operatorname{Sing}(D)$ also verifies $P \in$ $\operatorname{Sing}(D) \cap \mathcal{Z}\left(f_{d+k}\right)$.

The strict transform $\widetilde{V}$ of $V$ is nonsingular if and only if one of the following situations happens. Either $D \subset \mathbb{P}^{n}$ is a non-singular hypersurface or $k=1$ and $\operatorname{Sing}(D) \cap \mathcal{Z}\left(f_{d+1}\right)=\emptyset$ (this was proved by I. Luengo in [15] in the case $n=2$ but his proof does not depend on the dimension). In both cases the set $\mathcal{A}$ is empty. It can deduced from the works of Lê $\mathrm{D}$. T. and $\mathrm{B}$. Teissier, [13], that $\operatorname{Suf(f)}$ is bounded by $d$ when $D$ is non-singular. Assume then that $D$ has isolated singularities, i.e. $\operatorname{Sing}(D) \neq \emptyset$. 
I. Luengo and the author in [16] studied the remaining cases where the set $\mathcal{A}$ is empty. Thus if $\operatorname{Sing}(D) \cap \mathcal{Z}\left(f_{d+k}\right)=\emptyset$ then we computed explicitly all the polar quotients and in particular we got that $\operatorname{Suf}(\mathrm{f})$ is $d+k-1$ in this case.

In Theorem 1 we extend the above results and will prove that under condition $(*)$ then $\operatorname{Suf}(\mathbf{f})$ is bounded by

$$
d+k-1+\sup \{\mu(\tilde{V}, \tilde{P})-(k-1) \mu(D, P): \tilde{P} \in \mathcal{A}\},
$$

where $\mu(\tilde{V}, \tilde{P})$ and $\mu(D, P)$ are the Milnor numbers of $\widetilde{V}$ at $\widetilde{P}$, respect. of $D$ at the associated point $P$.

In Example 3 we apply our results to deal with the case where the projectivized tangent cone $D$ has at most non-degenerate critical points. We also compute explicitly the set of polar quotients and thus we get the value of $\operatorname{Suf}(f)$.

In Theorem 2 a Noether type formula for the Milnor number $\mu(V, 0)$ is given. This formula expresses $\mu(V, 0)$ in terms of the multiplicity of $V$ at 0 , the sum of the local Milnor numbers of $D$ at its singular points and the sum of the local Milnor numbers of $\widetilde{V}$ at its singular points.

Last section of the paper is devoted to the study of deformations of a hypersurface singularity $(V, 0)$ which satisfies condition $(*)$. We show in Theorem 3 that any deformation $\left(V_{t}, 0\right)$ of $(V, 0)$ with constant multiplicity and Milnor number is an equisingular deformation. Finally necessary and sufficient conditions for a deformation of $(V, 0)$ to be equisingular are given in Theorem 4. This result extends the same result proved by I. Luengo [15] in the case of superisolated surface singularities.

Notations. - Let $h \in \mathbb{C}\left[z_{0}, \ldots, z_{n}\right]$ be a homogeneous polynomial, we will denote by $\mathcal{Z}(h) \subset \mathbb{P}^{n}$ the divisor in $\mathbb{P}^{n}$ defined by $h$, and by $\operatorname{Sing}(\mathcal{Z}(h))$ its singular locus. If $P \in \operatorname{Sing}(\mathcal{Z}(h))$ is an isolated singularity of $\mathcal{Z}(h)$ then $\mu(\mathcal{Z}(h), P)$ will denote its Milnor number.

For the germ of a curve $(\gamma, 0) \subset\left(\mathbb{C}^{n+1}, 0\right)$ we denote the multiplicity of $\gamma$ at the origin by mult ${ }_{0}(\gamma)$. Let $(S, 0) \subset\left(\mathbb{C}^{n+1}, 0\right)$ be the germ of a hypersurface such that $\gamma$ is not contained in $S$, the intersection multiplicity of both germs at 0 will be denoted by $(S, \gamma)_{0}$.

\section{Decomposition of the polar curve}

Let $(V, 0) \subset\left(\mathbb{C}^{n+1}, 0\right)$ be the germ of a hypersurface. Throughout the paper $(V, 0)$ satisfies the condition: 
$(*)(V, 0)$ has an isolated singular point at 0 and the projectivized tangent cone of $V$ at 0 has at most isolated singularities.

It means that if $f$ is a holomorphic function such that $(V, 0)$ is its zero locus and if $f=f_{d}+f_{d+k}+\cdots$ is the Taylor expansion of $f$ around the origin then $D:=\mathcal{Z}\left(f_{d}\right) \subset \mathbb{P}^{n}$ is a projective hypersurface with at most isolated singularities.

Let $f: U \rightarrow \mathbb{C}$ be the germ of a holomorphic function such that its zero locus $(V, 0)$ has an isolated singularity. Let $H$ be a hyperplane in $\mathbb{C}^{n+1}$ passing through the origin. B. Teissier, [20] [21], introduced the notion of polar curve $\Gamma_{H}$ associated to $f$ and to $H$, the polar curve is the closure of the set

$$
\left\{p \in U \backslash\{0\}: T_{p} V_{p} \text { is parallel to } H\right\}
$$

where $V_{p}$ is the level hypersurface $f(z)-f(p)=0$ and $T_{p} V_{p}$ is its tangent space at $p$.

In fact, B. Teissier [20] [21] and J.P. Henry-M. Merle [8] proved that there exists a non-empty Zariski open subset $W_{1}$ in the Grassmannian of hyperplanes of $\mathbb{C}^{n+1}$ passing through 0 such that if $H \in W_{1}$ then $\Gamma_{H}$ is the germ of an isolated complete intersection singularity, transverse to $H$ and such that $\operatorname{mult}_{0}\left(\Gamma_{H}\right)=\left(\Gamma_{H}, H\right)_{0}=\mu^{(n)}(V, 0)$, where $\mu^{n}(V, 0)$ is the Milnor number of the intersection of $(V, 0)$ with a generic hyperplane. Moreover, the number of irreducible components (or branches) of $\Gamma_{H}$ is the same for all $H \in W_{1}$. Let $\Gamma_{H}=\bigcup_{i=1}^{r} \gamma_{q}$ be the decomposition of $\Gamma_{H}$ in branches. B. Teissier also proved that the sequence of multiplicities $m_{q}=\operatorname{mult}_{0}\left(\gamma_{q}\right)$ and the sequence of intersection multiplicities $\left(\gamma_{q}, V\right)_{0}$ do not depend on $H \in W_{1}$. Therefore, for each $q \in\{1, \ldots, r\}$, he defined two positive integers $\left(e_{q}, m_{q}\right): m_{q}=\operatorname{mult}_{0}\left(\gamma_{q}\right)$ and $e_{q}=\left(\gamma_{q}, V\right)_{0}-m_{q}$, and showed that

$$
\sum_{q=1}^{r} e_{q}=\mu(V, 0) \text { and } \sum_{q=1}^{r} m_{q}=\mu^{(n)}(V, 0) .
$$

Remark 1. - Let $(V, 0)$ be the germ of a hypersurface satisfying condition $(*)$. Since $D$ has a finite number of singular points there exists another non-empty Zariski open subset $W_{2}$ of hyperplanes such that $H \in W_{2}$ if and only if the projective variety $H \cap D$ is nonsingular. The condition (*), i.e. the finiteness of $\operatorname{Sing}(D)$, is the key point here because otherwise $\operatorname{Sing}(D)$ has dimension bigger than zero and any hyperplane always intersects $\operatorname{Sing}(D)$. Let $W:=W_{1} \cap W_{2}$. A hyperplane $H \in W$ will be called a good hyperplane for $(V, 0)$. 
Let $H \in W$ be a good hyperplane. We may choose coordinates so that the equation of $H$ is $\left\{z_{0}=0\right\}$. Let us denote $\frac{\partial f}{\partial z_{i}}$ by $f_{z_{i}}$. Thus $\Gamma_{H}$ is defined by $f_{z_{1}}=0, \ldots, f_{z_{n}}=0$ and $\operatorname{Sing}(D) \cap \mathcal{Z}\left(z_{0}\right)$ is empty. Let $C \Gamma_{H}$ be the projectivized tangent cone of $\Gamma_{H}$ at the origin.

Condition (*), i.e. the set $\operatorname{Sing}(D)$ is a finite set, is the key hypothesis of Proposition 1 in [16]. It was shown there that condition $(*)$ implies that $C \Gamma_{H}$ is the finite number of points $\left\{P_{1}, \ldots, P_{l}, P_{l+1}, \ldots, P_{s}\right\}$ given by the projective set $\mathcal{Z}\left(\left(f_{d}\right)_{z_{1}}, \ldots,\left(f_{d}\right)_{z_{n}}\right)$. We assume that $P_{i} \in \operatorname{Sing}(D)$ if $i=$ $1, \ldots, l$ and $P_{i} \notin \operatorname{Sing}(D)$ otherwise. Therefore, tangent line directions of branches of $\Gamma_{H}$ can be seen as points on $C \Gamma_{H}$. Let $P \in C \Gamma_{H}$ be such a point. We define $\Gamma_{P}$ as the union of branches of $\Gamma_{H}$ whose tangent line direction at the origin is defined by $P$. Then

$$
\Gamma_{H}=\bigcup_{P \in \operatorname{Sing}(D)} \Gamma_{P} \cup \bigcup_{P \notin \operatorname{Sing}(D)} \Gamma_{P} .
$$

Note that $\mathcal{Z}\left(\left(f_{d}\right)_{z_{1}}, \ldots,\left(f_{d}\right)_{z_{n}}\right)$ and $\mathcal{Z}\left(z_{0}\right)$ do not intersect because $\Gamma_{H}$ is transverse to $H$. Thus the tangent line directions of $\Gamma_{H}$ are not contained in $H$. The following lemma can also be found in [16].

Lemma 1. - For every $P \in C \Gamma_{H}$, the multiplicity of $\Gamma_{P}$ at the origin is equal to the intersection multiplicity of the projective hypersurfaces defined by the equations $\left(f_{d}\right)_{z_{1}}=0, \ldots,\left(f_{d}\right)_{z_{n}}=0$ at $P$. Therefore, if $P \in \operatorname{Sing}(D)$ then mult $_{0}\left(\Gamma_{P}\right)=\mu(D, P)$.

Remark 2. - If $D$ has an $A_{1}$-singularity at $P$, i.e. a non-degenerate singular point, then $\Gamma_{P}$ has only one smooth branch.

\section{A bound for the polar quotients}

Let $\pi:(\mathcal{X}, E) \rightarrow\left(\mathbb{C}^{n+1}, 0\right)$ be the blow-up of the origin; $(\tilde{V}, D)$ the strict transform of $(V, 0)$ and $E$ the exceptional divisor of $\pi$. From now on we are identifying points $P$ on $D$ with those $\tilde{P}$ on $\widetilde{V} \cap E$.

Lemma 2. - If $(V, 0)$ satisfies condition $(*)$ then the hypersurface $\tilde{V} \subset$ $\mathcal{X}$ has at most isolated singularities.

Proof. - Note that the singular locus of $\widetilde{V}$ is contained in $\operatorname{Sing}(D)$ which is a finite set. In order to prove it we look at a local equation of $\widetilde{V}$ at every point of $\widetilde{V} \cap E$.

Lemma 2 implies that every singular point $\widetilde{P}$ of $\widetilde{V}$ is related in a unique way with a point of $\operatorname{Sing}(D)$ (denoted by $P$ ). Let $\mathcal{A}$ be the set of points 
$\widetilde{P}$ of $\widetilde{V}$ such that $\widetilde{P} \in \operatorname{Sing}(\widetilde{V})$ and the associated point $P \in \operatorname{Sing}(D)$ also verifies $P \in \operatorname{Sing}(D) \cap \mathcal{Z}\left(f_{d+k}\right)$. Let $\tilde{P}$ be such an isolated singularity of $\widetilde{V}$ and $\mu(\tilde{V}, \tilde{P})$ its the Milnor number.

THEOREM 1. - Let $(V, 0)$ be the germ of a hypersurface satisfying the condition $(*)$ and $f=0$ an equation for $(V, 0)$. Let $f=f_{d}+f_{d+k}+\ldots$ be the Taylor expansion of $f$ around the origin. Then the supremum of the polar quotients $\sup \left\{\frac{e_{q}}{m_{q}}\right\}$ is less than or equal to

$$
d+k-1+\sup \{\mu(\tilde{V}, \tilde{P})-(k-1) \mu(D, P): \tilde{P} \in \mathcal{A}\} .
$$

From the proof of the above theorem we will show that for every $\tilde{P} \in \mathcal{A}$ the integer $\mu(\tilde{V}, \tilde{P})-(k-1) \mu(D, P)$ is non negative.

Proof. - Consider the open set $W$ of good hyperplanes for $(V, 0)$. Let $H \in W$, and $\Gamma_{H}=\bigcup_{q=1}^{r} \gamma_{q}$ be the polar curve. After a linear change of coordinates we suppose that $H$ is defined by $z_{0}=0$. Thus, $\Gamma_{H}$ has a decomposition as in the above section: $\Gamma_{H}=\cup \Gamma_{P}, P \in \mathcal{Z}\left(\left(f_{d}\right)_{z_{1}}, \ldots,\left(f_{d}\right)_{z_{n}}\right)$ and $\Gamma_{P}=\bigcup_{q=1}^{s} \gamma_{q}^{P}$, where $\gamma_{q}^{P}$ is a branch whose tangent line direction is defined by $P$, (we are using explicitly condition $(*)$ in order to have such a decomposition).

Let $P \in \mathcal{Z}\left(\left(f_{d}\right)_{z_{1}}, \ldots,\left(f_{d}\right)_{z_{n}}\right)$. Since $\Gamma_{H}$ is transverse to $H$, there is no tangent line direction of $\Gamma_{H}$ contained in $H$. Thus $P$ has homogeneous coordinates $\left(1: a_{1}: \ldots: a_{n}\right)$. Let $\psi: A:=\mathbb{C}^{n+1} \rightarrow B:=\mathbb{C}^{n+1}$ be the linear change of coordinates given by the equations $\tilde{z}_{0}=z_{0}$ and $\tilde{z}_{i}=z_{i}-a_{i} z_{0}$. Let $\varphi$ be its inverse morphism and $g:=f \circ \varphi=0$. Partial derivatives of $f$ and $g$ are related in the following way:

$$
g_{\tilde{z}_{0}}=f_{z_{0}} \circ \varphi+\sum_{i=1}^{n} a_{i} f_{z_{i}} \circ \varphi \text { and } g_{\tilde{z}_{i}}=f_{z_{i}} \circ \varphi .
$$

Thus, we find that:

(i) The Taylor expansion $g_{d}+g_{d+k}+\ldots$ of $g$ around the origin verifies $g_{s}=f_{s} \circ \varphi$. Therefore, $D$ is defined by $g_{d}=0$ and $g_{d+k}$ is different from zero.

(ii) $H$ is defined by $\tilde{z}_{0}=0$ and $Q:=\psi(P)$ has coordinates $(1,0, \ldots, 0)$.

(iii) The polar curve $\Gamma_{H}$ is defined by the zero locus of $g_{\tilde{z}_{i}}, i=1, \ldots, n$, and it is transverse to $H$. 
(iv) Let $I^{B}$ be the ideal $\left(g_{\tilde{z}_{1}}, \ldots, g_{\tilde{z}_{n}}\right) \mathbb{C}\left\{\tilde{z}_{0}, \ldots, \tilde{z}_{n}\right\}$. The ideal of initial forms of $I^{B}$ is generated by $\left(g_{d}\right) \tilde{z}_{i}, i \in\{1, \ldots, n\}$.

Take $\gamma_{q}^{P}$ to be a branch of $\Gamma_{P}$. Let $m_{q}=\operatorname{mult}_{0}\left(\gamma_{q}^{P}\right)$ and $e_{q}=\left(\gamma_{q}^{P}, V\right)_{0}-$ $m_{q}$, Since $(1: 0: \ldots: 0)$ is the tangent line direction of $\gamma_{q}^{P}$ in $B, \gamma_{q}^{P}$ has a parametrization $h:(\mathbb{C}, 0) \rightarrow\left(\mathbb{C}^{n+1}, 0\right): h(t)=\left(t^{m_{q}}, h_{1}(t), \ldots, h_{n}(t)\right)$, where $\operatorname{ord}_{t}\left(h_{i}^{B}(t)\right)>m_{q}$ for all $i \in\{1, \ldots, n\}$. Of course $\varphi \circ h$ is a parametrization of the germ $\gamma_{q}^{P}$ in $A$.

One checks using the Chain Rule that $e_{q}=\left(\gamma_{q}^{P}, S\right)_{0}$, where $(S, 0)$ is the germ of hypersurface defined by $\left\{f_{z_{0}}=0\right\}$ in $A$ and by $\left\{f_{z_{0}} \circ \varphi=0\right\}$ in $B$. Now we have two possibilities:

1. Suppose that $\left(f_{d}\right)_{z_{0}}$ is different from zero, then we have the following two possibilities:

(A) If $P \notin \operatorname{Sing}(D)$ then $\gamma_{q}^{P}$ is transverse to $(S, 0)$ at 0 because $P$ is not on $\mathcal{Z}\left(\left(f_{d}\right)_{z_{0}}\right)$. Hence, the intersection multiplicity of both germs at the origin is the product of their multiplicities: $e_{q}=\left(\gamma_{q}^{P}, S\right)_{0}=\operatorname{mult}_{0}\left(\gamma_{q}^{P}\right)$. $\operatorname{mult}_{0}(S)=m_{q}(d-1)$. Therefore, for every branch $\gamma_{q}^{P}$ of $\Gamma_{H}$ such that $P \notin \operatorname{Sing}(D)$, we get the equality

$$
\frac{e_{q}}{m_{q}}=d-1<d=\operatorname{mult}_{0}(V) .
$$

Remark 3. - In particular if the projectivized tangent cone $D \subset \mathbb{P}^{n}$ is nonsingular then the all the polar quotients of the isolated singularity $(V, 0)$ are equal to $d-1$, where $d$ is the multiplicity of $V$ at 0 . Then the set $\left\{\frac{e_{q}}{m_{q}}\right\}$ is nothing but $\{d-1\}$ and $\operatorname{Suf}(\mathrm{f})=d$.

(B) Let $P \in \operatorname{Sing}(D)$. If $P \notin \mathcal{Z}\left(f_{d+k}\right)$ then we have proved in [16] Theorem 2 that in this case the polar quotient $\frac{e_{q}}{m_{q}}=d+k-1$. Then let us assume that $P \in \operatorname{Sing}(D) \cap \mathcal{Z}\left(f_{d+k}\right)$. Let $\pi: \mathcal{B} \rightarrow B$ be the blowup centered at the origin. Since $Q$ has coordinates $(1,0, \ldots, 0)$, the strict transform $\widetilde{\gamma}_{q}^{P}$ of $\gamma_{q}^{P}$ lies into the chart where $\pi$ is given by $\pi\left(x_{0}, \ldots, x_{n}\right)=$ $\left(x_{0}, x_{1} x_{0}, \ldots, x_{n} x_{0}\right)$. By [1] p. 32, a parametrization of $\widetilde{\gamma}_{q}^{P}$ is given by $x_{0}=$ $t^{m_{q}}$ and $x_{i}=\frac{h_{i}(t)}{t^{m q}}, i=1 \ldots, n$. Moreover $g \circ \pi$ is equal to $x_{0}^{d} \cdot \tilde{g}\left(x_{0}, \ldots, x_{n}\right)$ where

$$
\tilde{g}=g_{d}\left(1, x_{1}, \ldots, x_{n}\right)+x_{0}^{k} m\left(x_{0}, \ldots, x_{n}\right)=0
$$

is a local equation of $\widetilde{V}$ at $\tilde{Q}$ and $m \in \mathbb{C}\left\{x_{0}, \ldots, x_{n}\right\}$ holds $m(0)=0$. By (iv) and Proposition 13.13 in [9], the strict transform $\tilde{\Gamma}_{H}$ of $\Gamma_{H}$ by $\pi$ is the 
zero locus of $\widetilde{g_{\tilde{z}}}, \ldots, \widetilde{g_{z_{n}}}$ with

$$
x_{0}^{d} \cdot \widetilde{g_{z_{i}}}\left(x_{0}, \ldots, x_{n}\right)=g_{\tilde{z}_{i}}\left(x_{0}, x_{1} x_{0}, \ldots, x_{n} x_{0}\right) .
$$

It is easy to check, for every $i \in\{1, \ldots, n\}$, that $(\tilde{g})_{x_{i}}=\widetilde{g_{z_{i}}}$. Then $\tilde{\Gamma}_{H}$ is the zero locus of $(\tilde{g})_{x_{1}}, \ldots,(\tilde{g})_{x_{n}}$. Moreover, since $\widetilde{\gamma}_{q}^{P}$ is a branch of $\tilde{\Gamma}_{H}$, for every $i \in\{1, \ldots, n\}$, we find that $(\tilde{g})_{x_{i}} \circ \tilde{h}_{q}(t) \equiv 0$. This implies the following equalities:

$$
\begin{aligned}
\operatorname{ord}_{t}(\tilde{g} \circ \tilde{h}) & =\operatorname{ord}_{t}\left(\frac{\partial(\tilde{g} \circ \tilde{h})}{\partial t}\right)+1 \\
& =\operatorname{ord}_{t}\left(\left((\tilde{g})_{x_{0}} \circ \tilde{h}\right) \cdot \frac{\partial\left(t^{m_{q}}\right)}{\partial t}\right)+1 \\
& =\operatorname{ord}_{t}\left(\left((\tilde{g})_{x_{0}} \circ \tilde{h}\right)\right)+m_{q} .
\end{aligned}
$$

Therefore the sum of the polar invariants $e_{q}$ and $m_{q}$ satisfies:

$$
\begin{aligned}
e_{q}+m_{q} & =\left(\gamma_{q}^{P}, V\right)_{0}=\operatorname{ord}_{t}(g \circ h)=\operatorname{ord}_{t}(g \circ \pi \circ \tilde{h}) \\
& =\operatorname{ord}_{t}\left(\left(x_{0}^{d} \cdot \tilde{g}\right) \circ \tilde{h}\right)=d m_{q}+\operatorname{ord}_{t}(\tilde{g} \circ \tilde{h}) \\
& =(d+1) m_{q}+\operatorname{ord}_{t}\left((\tilde{g})_{x_{0}} \circ \tilde{h}\right) .
\end{aligned}
$$

Let us compute $(\tilde{g})_{x_{0}}$. From its definition $(\tilde{g})_{x_{0}}$ is equal to $x_{0}^{k-1} \cdot s, s \in$ $\mathbb{C}\left\{x_{0}, \ldots, x_{n}\right\}$ with $s(0)=0$. Let $A_{q}^{P}:=\operatorname{ord}_{t}(s \circ \tilde{h})>0$, then

$$
e_{q}=(d+k-1) \cdot m_{q}+A_{q}^{P}
$$

Thus we find the following equality for the quotient of the polar invariants:

$$
\frac{e_{q}}{m_{q}}=d+k-1+\frac{A_{q}^{P}}{m_{q}}
$$

By the other hand, Lemma 2 shows that $\widetilde{V}$ has an isolated singularity at $\tilde{P}$ or $\tilde{Q}$. A local equation of $\widetilde{V}$ at $\tilde{Q}$ is $\tilde{g}=0$, therefore the Milnor number of $\widetilde{V}$ at $\tilde{P}$ is given by

$$
\mu(\tilde{V}, \tilde{P})=\mu(\widetilde{V}, \tilde{Q})=\operatorname{dim}_{\mathbb{C}} \frac{\mathbb{C}\left\{x_{0}, \ldots, x_{n}\right\}}{\left(\tilde{g}_{x_{0}}, \ldots, \tilde{g}_{x_{n}}\right)} .
$$

Moreover, since $\tilde{\Gamma}_{H}$ is defined by $(\tilde{g})_{x_{1}}, \ldots,(\tilde{g})_{x_{n}}$ we obtain that the above Milnor number is

$$
\mu(\tilde{V}, \tilde{P})=\left(\left\{(\tilde{g})_{x_{0}}=0\right\}, \tilde{\Gamma}_{H}\right)_{0}=\sum_{\gamma_{q}^{P}} \operatorname{ord}_{t}\left((\tilde{g})_{x_{0}} \circ \tilde{h}\right) .
$$


Lemma 1 and our previous computations show the relations:

$$
\mu(\tilde{V}, \tilde{P})=\sum_{\gamma_{q}^{P}}\left((k-1) m_{q}+A_{q}^{P}\right)=(k-1) \mu(D, P)+\sum_{\gamma_{q}^{P}} A_{q}^{P} .
$$

Note that $\mu(\tilde{V}, \tilde{P})-(k-1) \mu(D, P)$ is a positive integer because every $A_{q}^{P}$ is positive too. Thus we get the following bound from (6):

$$
\frac{e_{q}}{m_{q}} \leqslant d+k-1+\mu(\tilde{V}, \tilde{P})-(k-1) \mu(D, P) .
$$

Remark 4. - Note that if $\widetilde{P}$ is a nonsingular point of $\tilde{V}$ then $\mu(\tilde{V}, \tilde{P})=0$ and from (8) we get $k=1$ and $A_{q}^{P}=0$ for every $q$. It implies after (6) that $\frac{e_{q}}{m_{q}}=d$. This coincides with the following fact already noted by I. Luengo in [15]: the isolated hypersurface singularity $(V, 0)$ which is not a cone can be resolved blowing-up once the origin if and only if $k=1$ and $\operatorname{Sing}(D) \cap \mathcal{Z}\left(f_{d+1}\right)=\emptyset$.

2. If $\left(f_{d}\right)_{z_{0}} \equiv 0$ then $\operatorname{Sing}(D)=\mathcal{Z}\left(\left(f_{d}\right)_{z_{1}}, \ldots,\left(f_{d}\right)_{z_{n}}\right)=\{P=(1: 0$ : $\ldots: 0)\}$ and we get the same result as in (b). Here we are using condition (*) because we are using our decomposition of the polar curve.

COROLlaRY 1. - Let $H$ be a good hyperplane for an isolated hypersurface singularity $(V, 0) \subset\left(\mathbb{C}^{n+1}, 0\right)$ verifying condition $(*)$. Then the singularity $(V \cap H, 0)$ can be deformed toward its tangent cone $(D \cap H, 0)$ in a $\mu^{*}$-constant deformation and for every $i \in\{1, \ldots, n\}$, the Milnor number of the general $i$-plane section is $\mu^{(i)}(V \cap H, 0)=\mu^{(i)}(D \cap H, 0)=(d-1)^{i}$.

Proof. - Since $H$ is a good hyperplane for $(V, 0)$ then $D \cap H$ is a nonsingular hypersurface in $\mathbb{P}^{n-1}$. If $\left\{z_{0}=0\right\}$ is an equation for $H$ then the singularity $(V \cap H, 0) \subset\left(\mathbb{C}^{n}, 0\right)$ is defined by $f\left(0, z_{1}, z_{2}, \ldots, z_{n}\right)=0$ and its projectivized tangent cone $D \cap H$ (defined by $f_{d}\left(0, z_{1}, z_{2}, \ldots, z_{n}\right)=0$ ) is nonsingular. It implies, after Remark 3 , that $(V \cap H, 0) \subset\left(\mathbb{C}^{n}, 0\right)$ is topologically equivalent to the singularity defined by its tangent cone. It turns out, after B. Teissier's results (see Introduction) that, for every $i \in\{1, \ldots, n\}$, we find that $\mu^{(i)}(V \cap H, 0)=\mu^{(i)}(D \cap H, 0)=(d-1)^{i}$.

Example 1. - Let $f=f_{d}+f_{d+k}:\left(\mathbb{C}^{3}, 0\right) \rightarrow(\mathbb{C}, 0)$ be a germ of holomorphic function such that its zero locus $(V, 0)$ satisfies the condition $(*)$. It is clear that in this case the set $\operatorname{Sing}(D) \cap \operatorname{Sing}\left(\mathcal{Z}\left(f_{d+k}\right)\right)$ is empty. Moreover, for every $\tilde{P} \in \operatorname{Sing}(\tilde{V})$ such that $P \in \operatorname{Sing}(D) \cap \mathcal{Z}\left(f_{d+k}\right)$ one can compute (see the local equation of $\widetilde{V}$ at $\tilde{P}$ after blows up the origin) the Milnor number

$$
\begin{gathered}
\mu(\tilde{V}, \tilde{P})=(k-1) \mu(D, P)+k\left(s_{P}-1\right) \\
-680-
\end{gathered}
$$


where $s_{P}$ is the intersection multiplicity of the projective plane curves $D$ and $\mathcal{Z}\left(f_{d+k}\right)$ at $P$. Therefore, in this case our bound is equivalent to the following one

$$
\sup \left\{\frac{e_{q}}{m_{q}}\right\} \leqslant d+k-1+\sup \left\{k\left(s_{P}-1\right): \quad \tilde{P} \in \mathcal{A}\right\} .
$$

Example 2. - Let $f=\left(x^{3} y^{2}+z^{5}\right)\left(z^{2}+y x\right)+z x^{8}+z y^{8}+x^{6} y^{3}+z^{14} x^{15}$ and $(V, 0)$ its zero locus which satisfies the condition $(*)$. If we apply Example 1 to the singularity defined by $g=\left(x^{3} y^{2}+z^{5}\right)\left(z^{2}+y x\right)+z x^{8}+z y^{8}+x^{6} y^{3}$ we get that for $g$ the supremum of the polar quotients is bounded by 12. Then $f$ and $g$ are topologically equivalents.

Example 3. - Suppose that $D$ has only $A_{1}$-singularities, i.e. all singular points of $D$ are non-degenerate. Let $H$ be a good hyperplane for $(V, 0)$ and we may assume that it has equation $z_{0}=0$. Note that $\left(f_{d}\right)_{z_{0}} \not \equiv 0$, otherwise Sing $(D)=\{P=(1: 0: \ldots: 0)\}$ and $D$ defines a smooth hypersurface in $\mathbb{P}^{n-1}$ which is in contradiction with the fact that $P$ is an $A_{1}$-singularity. Let $\Gamma_{H}=\cup \Gamma_{P}, P \in \mathcal{Z}\left(\left(f_{d}\right)_{z_{1}}, \ldots,\left(f_{d}\right)_{z_{n}}\right)$ be our decomposition of the polar curve. We have proved into the proof of Theorem 1 that:

1. If $P \notin \operatorname{Sing}(D)$ then for each branch $\gamma_{q}$ of $\Gamma_{P}$ the quotient $\frac{e_{q}}{m_{q}}=d-1$, see (2).

2. If $P \in \operatorname{Sing}(D)$ then Remark 2 shows that $\Gamma_{P}$ consists only on one branch with multiplicity one. Furthermore the equalities (6) and (8) give $\frac{e_{q}}{m_{q}}=d+\mu(\tilde{V}, \tilde{P})$.

Thus, we obtain that the set of polar quotients is the following set:

$$
\left\{\frac{e_{q}}{m_{q}}\right\}_{r e d}=\{d-1, d+\mu(\widetilde{V}, \tilde{P})\}_{P \in \operatorname{Sing}(D)} .
$$

Moreover in this case it is possible to describe the jacobian Newton polygon $\sum_{q=1}^{r} \frac{e_{q}}{\overline{m_{q}}}$ of the image of $\Gamma_{H}$ by the map $\phi: \mathbb{C}^{n+1} \rightarrow \mathbb{C}^{2}$ defined by $\phi(\bar{z})=\left(f_{z_{0}}(\bar{z}), z_{0}\right)$, see [21] [22]. Note that this polygon is a (c)-equisingularity invariant, see [21]. Let $P_{1}, \ldots, P_{s}$ be the singular points of $D$. Suppose that they are ordered as follows: $P_{i} \leqslant P_{j}$ if and only if $\mu\left(\tilde{V}, \tilde{P}_{i}\right) \leqslant \mu\left(\tilde{V}, \tilde{P}_{j}\right)$. Since each singularity of $D$ is an $A_{1}$-singularity then the sum of Milnor numbers of $D$ at all singular points is equal to $s$. Lemma 
2 and $(a)$ into the proof of Theorem 1 show that the bellow defined integers $M$ and $N$ satisfying the following equalities:

$$
\begin{gathered}
M:=\sum_{P \notin \operatorname{Sing}(D)} \sum_{\gamma_{q}^{P}} m_{q}=(d-1)^{n}-s \text { and } \\
N:=\sum_{P \notin \operatorname{Sing}(D)} \sum_{\gamma_{q}^{P}} e_{q}=(d-1)^{n+1}-(d-1) s .
\end{gathered}
$$

Then the jacobian Newton polygon of $(V, 0)$ has the following form:

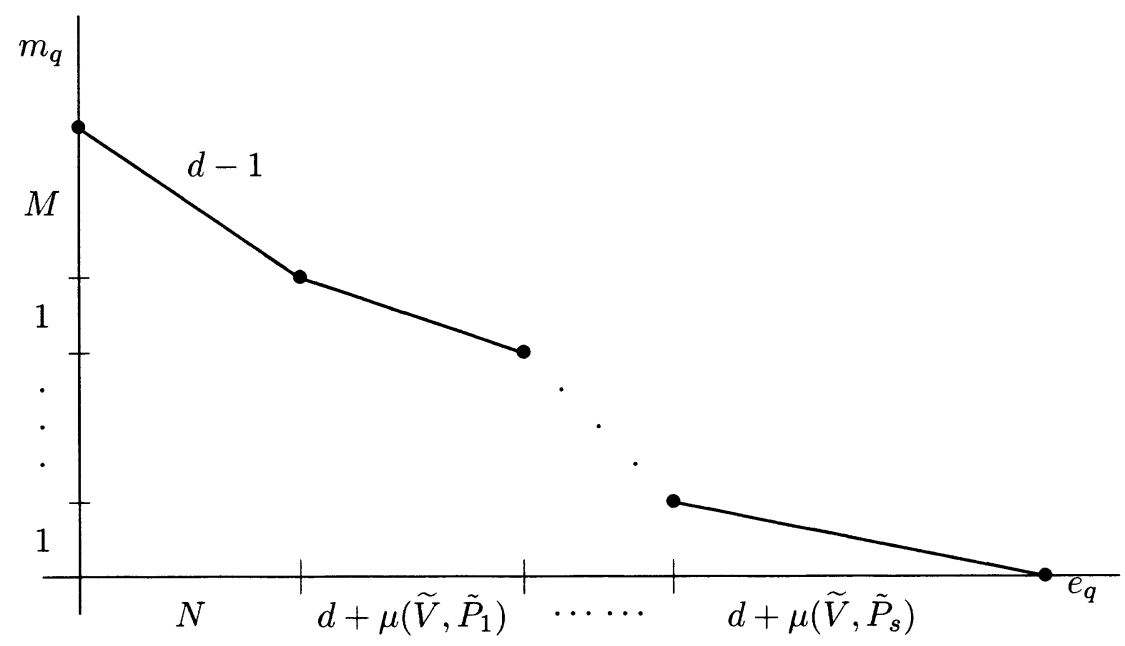

Next theorem gives a Noether type formula for the Milnor number $\mu(V, 0)$. This formula is a relation among the Milnor number of $(V, 0)$, its multiplicity at 0 , the sum of the Milnor numbers of $D$ at its singular points; it is denoted by $\mu(D)$ and the sum of the Milnor numbers of $\widetilde{V}$ at its singular points, which will be denoted by $\mu(\widetilde{V})$. Notice that in our hypothesis both $\mu(D)$ and $\mu(\widetilde{V})$ are finite numbers. In fact, the same formula has been generalized by the author in [17] to any isolated hypersurface singularity $(V, 0) \subset\left(\mathbb{C}^{n+1}, 0\right)$.

THEOREM 2. - If $(V, 0)$ satisfies condition $(*)$ then

$$
\mu(V, 0)=(d-1)^{n+1}+\mu(D)+\mu(\widetilde{V}) .
$$

Proof. - Let $f=f_{d}+f_{d+1}+\ldots \in \mathbb{C}\left\{z_{0}, \ldots, z_{n}\right\}$ be the germ of a holomorphic function such that its zero locus defines $(V, 0)$. Let $W$ be 
the open set of good hyperplanes for $(V, 0)$ and $H \in W$. Suppose that $\left\{z_{0}=0\right\}$ defines $H$. Therefore $\Gamma_{H}=\cup \Gamma_{P}, P \in \mathcal{Z}\left(f_{d_{z_{1}}}, \ldots, f_{d_{z_{n}}}\right)$, is our decomposition of the polar curve where $\Gamma_{P}=\bigcup \gamma_{q}^{P}$. Corollary 1 and Lemma 1 show that:

$$
(d-1)^{n}=\mu^{(n)}(V, 0)=\sum_{q} m_{q}=\sum_{P \notin \operatorname{Sing}(D)} \operatorname{mult}_{0}\left(\Gamma_{P}\right)+\mu(D) .
$$

Hence, we find that

$$
\begin{aligned}
\mu(V, 0)=\sum e_{q} & =\sum_{P \notin \operatorname{Sing}(D)} \sum_{\gamma_{q}^{P}} e_{q}+\sum_{P \in \operatorname{Sing}(D)} \sum_{\gamma_{q}^{P}} e_{q} \\
& =\sum_{P \notin \operatorname{Sing}(D)} \sum_{\gamma_{q}^{P}}(d-1) m_{q}+\sum_{P \in \operatorname{Sing}(D)} \sum_{\gamma_{q}^{P}} e_{q} \\
& =(d-1)^{n+1}-(d-1) \sum_{P \in \operatorname{Sing}(D)} \mu(D, P)+\sum_{P \in \operatorname{Sing}(D)} \sum_{\gamma_{q}^{P}} e_{q} .
\end{aligned}
$$

Let $P \in \operatorname{Sing}(D)$, by Lemma $1,(4)$ and $(7)$, we obtain that

$$
\begin{aligned}
\sum_{\gamma_{q}^{P}} e_{q} & =\sum_{\gamma_{q}^{P}}\left(d \cdot m_{q}+\operatorname{ord}_{t}\left((\tilde{g})_{x_{0}} \circ \tilde{h}\right)\right) \\
& =d \cdot \sum_{\gamma_{q}^{P}} m_{q}+\sum_{\gamma_{q}^{P}} \operatorname{ord}_{t}\left((\tilde{g})_{x_{0}} \circ \tilde{h}\right) \\
& =d \cdot \mu(D, P)+\sum_{\gamma_{q}^{P}} \operatorname{ord}_{t}\left((\tilde{g})_{x_{0}} \circ \tilde{h}\right) \\
& =d \cdot \mu(D, P)+\mu(\widetilde{V}, \tilde{P}) .
\end{aligned}
$$

Substituting in (9) we get the proof of the theorem.

Remark 5. - The above formula is a generalization of the well-known Noether's formula for germs of plane curve singularities: $\mu(V, 0)=d(d-$ 1) $+\mu(\tilde{V})$.

\section{Multiplicity and $\mu$-constant deformations}

Throughout this paragraph $(V, 0) \subset\left(\mathbb{C}^{n+1}, 0\right)$ will denote the germ of a hypersurface satisfying condition $(*)$. Assume that $(V, 0)$ has multiplicity $d$ at 0 . In this section we deal with $\mu$-constant deformations of $(V, 0)$.

First we recall some concepts on deformations, we follow Chapter 6 in the book [14] of E. Looijenga or Chapter III in [20]. 
A deformation of an isolated hypersurface singularity $(V, 0)$ is a mapgerm $f:(X, x) \rightarrow(S, s)$ between nonsingular germs such that $(V, 0)$ is isomorphic to $\left(f^{-1}(s), x\right)$. The space $(S, s)$ is called the base space of the deformation. There exist an obvious way to define morphisms of deformations. Then a deformation is called versal if any other deformation factorized through it. The factorization morphism is not unique. Nevertheless there exist deformations such that the derivative map between the base spaces is unique. Such deformations are called miniversal deformations. Any isolated hypersurface singularity admits a miniversal deformation and any of two of such deformations are isomorphic. It is also known that the base space of the miniversal deformation of an isolated hypersurface singularity is $\left(\mathbb{C}^{\tau}, 0\right)$ where $\tau$ is the Tjurina number of $(V, 0)$.

Let $m:(\mathcal{M}, 0) \rightarrow\left(\mathbb{C}^{\tau}, 0\right)$ be the miniversal deformation of $(V, 0) \subset$ $\left(\mathbb{C}^{n+1}, 0\right)$. Let $\mathcal{M}_{a}$ be a sufficiently small representative of $m^{-1}(a)$. We set:

$$
\begin{array}{r}
S_{\mu, d}=\left\{a \in \mathbb{C}^{\tau}: \mathcal{M}_{a} \text { has a singular point with the same } \mu\right. \\
\text { and multiplicity as }(V, 0)\},
\end{array}
$$

$S_{\mu, \mu^{(n)}}=\left\{a \in \mathbb{C}^{\tau}: \mathcal{M}_{a}\right.$ has a singular point with the same $\mu$

$$
\text { and } \left.\mu^{(n)} \text { as }(V, 0)\right\}
$$

$S_{\mu^{*}}=\left\{a \in \mathbb{C}^{\tau}: \mathcal{M}_{a}\right.$ has a singular point with the same $\mu^{*}$ as $\left.(V, 0)\right\}$.

We will prove that $S_{\mu, d}=S_{\mu, \mu^{(n)}}=S_{\mu^{*}}$ and we give another description of this stratum. Let $\mathbb{D}=\{t \in \mathbb{C}:|t|<\varepsilon\}$.

TheOREM 3. - Let $p: \mathcal{B} \rightarrow \mathbb{D}$ be a $\mu$-constant deformation of $(V, 0)$. Let $\sigma: \mathbb{D} \rightarrow \mathcal{B}$ be the section of $p$ such that $\left(p^{-1}(t):=V_{t}, \sigma^{-1}(t)\right)$ has an isolated singularity whose Milnor number does not depend on $t,(V, 0)=$ $\left(p^{-1}(0), \sigma^{-1}(0)\right)$. The following conditions are equivalent:

(i) The deformation is $\mu^{(n)}$-constant.

(ii) The multiplicity of $V_{t}$ at $\sigma^{-1}(t)$ is constant.

(iii) The deformation is $\mu^{*}$-constant.

Proof. - Let us start with $(i)$ implies $(i i)$. Let $H$ be a general hyperplane for $(V, 0)$. For $t$ small enough $H$ is also a general hyperplane for $\left(V_{t}, \sigma^{-1}(t)\right)$. Since the projectivized tangent cone $D_{0} \subset \mathbb{P}^{n}$ of $(V, 0)$ at 0 defines a hypersurface with only isolated singularities then, after Corollary $1,(V \cap H, 0)$ can deformed in a $\mu^{*}$-constant deformation toward $\left(D_{0} \cap H, 0\right)$. From hypothesis (i) we get a $\mu$-constant deformation of $\left(V_{t} \cap H, \sigma^{-1}(t)\right)$ toward $\left(D_{0} \cap H, 0\right)$. 
The isolated singularity $\left(D_{0} \cap H, 0\right)$ is a cone which is homogeneous. For such homogeneous singularities it is known that $\mu$-constant deformations implies "multiplicity constant" deformations, see [7] [18]. It implies that for every $t \in \mathbb{D}$ the multiplicity of $V_{t} \cap H$ at $\sigma^{-1}(t)$ ) is constant. To finish the proof it is enough to note that since $H$ is a generic hyperplane the multiplicity of $\left(V_{t} \cap H\right.$ at $\left.\sigma^{-1}(t)\right)$ is equal to the multiplicity of $V_{t}$ at $\left.\sigma^{-1}(t)\right)$.

Next we prove (ii) implies (iii).

Suppose that the multiplicity is constant. After an analytic change of coordinates we may assume that $\sigma(t)=(0, \ldots, 0, t)$, i.e. $\sigma(\mathbb{D})$ is defined by $z_{0}=0, \ldots, z_{n}=0$. Let $F(\bar{z}, t)=0$ be the equation of $\mathcal{B}$ and $p(\bar{z}, t)=t$. Since the multiplicity is constant, we can write $F$ as follows:

$$
F(\bar{z}, t)=F_{d}(\bar{z}, t)+F_{d+1}(\bar{z}, t)+\ldots
$$

where each $F_{i} \in \mathbb{C}\{t\}[\bar{z}]$ is a homogeneous polynomial of degree $i$. The equation $F_{d}(\bar{z}, t)=0$ defines a family $\mathcal{D}=\left\{D_{t}\right\}_{t \in \mathbb{D}}$ of projective hypersurfaces of degree $d$ in $\mathbb{P}^{n}$. In order to show (iii), it is enough to prove that any $D_{t} \subset \mathbb{P}^{n}$ has at most isolated singularities. After that we apply Remark 1 . Let $C$ be the analytic set of $\mathbb{P}^{n} \times \mathbb{D}$ defined by the zero locus of $\frac{\partial F_{d}}{\partial z_{0}}, \ldots, \frac{\partial F_{d}}{\partial z_{n}}$. The second projection restricted to $C$ gives a finite morphism between $C$ and $\mathbb{D}$ because the preimage over the origin is a discrete set, see e.g. [10] p. 164. Then, for all $t \in \mathbb{D}$, the set $D_{t} \cap P$ is a finite set, that means that $\operatorname{Sing}\left(D_{t}\right)$ is a finite set.

Finally (iii) implies (i) follows by definition.

Let $p: \mathcal{B} \rightarrow \mathbb{D}$ be a multiplicity constant deformation of $(V, 0)$ where $(V, 0)$ verifies condition $(*)$. Suppose that $F(\bar{z}, t)=0$ is an equation for $\mathcal{B}$ and $p(\bar{z}, t)=t$. Thus, $F(\bar{z}, t)=F_{d}(\bar{z}, t)+F_{d+1}(\bar{z}, t)+\ldots, F_{d}(\bar{z}, t)=0$ defines a family $\mathcal{D}=\left\{D_{t}\right\}_{t \in \mathbb{D}}$ of hypersurfaces in $\mathbb{P}^{n}$ such that $D_{0}$ has at most isolated singularities. Notice that in the above proof we have shown that every projective hypersurface $D_{t}$ has at most isolated singularities.

Since the multiplicity is constant, let $\pi: \widetilde{\mathcal{B}} \rightarrow \mathcal{B}$ be the monoidal transformation of $\mathcal{B}$ with center $\sigma^{-1}(\mathbb{D})$. Let $P$ be a singular point of $D$ and assume that it has homogeneous coordinates $(1: 0: \ldots: 0)$. Let $\tilde{P}$ be the corresponding point of $\widetilde{\mathcal{B}}$. In a neighbourhood of $\tilde{P}$ the map $\pi$ is given by $\pi\left(x_{0}, x_{1}, \ldots, x_{n}, t\right)=\left(x_{0}, x_{0} x_{1}, \ldots, x_{0} x_{n}, t\right)$ and a local equation of $\widetilde{\mathcal{B}}$ at $\tilde{P}$ is

$$
\tilde{F}\left(x_{0}, \ldots, x_{n}, t\right)=F_{d}\left(1, x_{1}, \ldots, x_{n}, t\right)+x_{0} \cdot \tilde{F}_{d+1}\left(1, x_{1}, \ldots, x_{n}, t\right)+\ldots
$$

Then $p \circ \pi:(\widetilde{\mathcal{B}}, \tilde{P}) \rightarrow(\mathbb{D}, 0)$ is a deformation of the germ of hypersurface with isolated singularity $(\tilde{V}, \tilde{P})$, see $(2)$. The hypersurface $(p \circ \pi)^{-1}(t)$ is the 
strict transform $\tilde{V}_{t}$ of $V_{t}$ after blowing-up the origin. The next theorem gives necessary and sufficient conditions for $p$ to be a $\mu^{*}$-constant deformation.

THEOREM 4.- Let $p: \mathcal{B} \rightarrow \mathbb{D}$ be a multiplicity constant deformation of $(V, 0)$ such that every $V_{t}:=p^{-1}(t)$ has isolated singularities. The deformation $p$ is a $\mu^{*}$-deformation if and only if the following conditions holds:

(a) For each singular point $P_{i} \in \operatorname{Sing}(D)$, there exists a (unique) section $\tau_{i}: \mathcal{T} \rightarrow \mathcal{D}$ such that $\tau_{i}(t) \in \operatorname{Sing}\left(D_{t}\right)$ and $\mu\left(D, P_{i}\right)=\mu\left(D_{t}, \tau_{i}(t)\right)$.

(b) For each singular point $P_{i} \in \operatorname{Sing}(D)$, there exists a (unique) section $\eta_{i}: \mathcal{T} \rightarrow \widetilde{\mathcal{B}}$ such that $\eta_{i}(t) \in \operatorname{Sing}\left(\tilde{V}_{t}\right)$ and $\mu\left(\tilde{V}, \tilde{P}_{i}\right)=\mu\left(\tilde{V}_{t}, \eta_{i}(t)\right)$.

Proof. - Suppose that $p: \mathcal{B} \rightarrow \mathbb{D}$ is a $\mu^{*}$-deformation. Thus there exists a section $\sigma: \mathbb{D} \rightarrow \mathcal{B}$ such that $\left\{\left(V_{t}, \sigma(t)\right)\right\}$ is a $\mu^{*}$-constant family. By the semicontinuity of the Milnor number, for every $P_{i} \in \operatorname{Sing}(D)$, there exist singular points $x_{i_{1}}(t), \ldots, x_{i_{k_{i}}}(t)$ of $D_{t}$ such that

$$
\mu\left(D, P_{i}\right) \geqslant \sum_{j=1}^{k_{i}} \mu\left(D_{t}, x_{i_{j}}(t)\right) .
$$

In the same way, for each $P_{i} \in \operatorname{Sing}\left(D_{0}\right)$, there exist singular points $y_{i_{1}}(t), \ldots, y_{i_{s_{i}}}(t)$ of $\tilde{V}_{t}$ such that

$$
\mu\left(\tilde{V}, \tilde{P}_{i}\right) \geqslant \sum_{l=1}^{s_{i}} \mu\left(\tilde{V}_{t}, y_{i_{l}}(t)\right) .
$$

Since the deformation is $\mu^{*}$-constant, the formula for the Milnor number in Theorem 2 gives the equality:

$$
\begin{aligned}
\sum_{P \in \operatorname{Sing}(D)} \mu(D, P) & +\sum_{P \in \operatorname{Sing}(D)} \mu(\tilde{V}, \tilde{P}) \\
& =\sum_{P_{t} \in \operatorname{Sing}\left(D_{t}\right)} \mu\left(D_{t}, P_{t}\right)+\sum_{P_{t} \in \operatorname{Sing}\left(D_{t}\right)} \mu\left(\tilde{V}_{t}, \tilde{P}_{t}\right) .
\end{aligned}
$$

Thus the inequalities (10) and (11) are equalities. Finally applying the irreducibility of the singular locus, see [11], we get the sections of the Theorem.

Next we prove the "if part". Assume that the conditions $(a)$ and $(b)$ holds. Since $D_{t}$ has at most isolated singularities and by the semicontinuity of the (local) Milnor number, there exists a bijection between the sets 
On polar invariants of hypersurface singularities

$\operatorname{Sing}(D)$ and $\operatorname{Sing}\left(D_{t}\right)$. This implies that $\mu(D)=\mu\left(D_{t}\right)$ and $\mu(\widetilde{V})=\mu\left(\tilde{V}_{t}\right)$. Next we apply the formula for the Milnor number in Theorem 2 and we get that the family $\left\{V_{t}\right\}$ is a $\mu$-constant family. Since by hypothesis it is a multiplicity constant family then Theorem 3 gives the result.

Acknowledgements. - This work was finished when the author was visiting the École Normale Supérieure in Paris. The author is very much thankful to E. García-Barroso, P. Popescu and B. Teissier for their helpful discussions on this topic and their hospitality.

\section{Bibliography}

[1] Campillo (A.). - Algebroid Curves in Positive Characteristic, Lecture Notes in Math., vol. 813, Springer-Verlag, Berlin-Heidelberg-New York, 1980.

[2] Casas-Alvero (E.). - 'Infinitely near imposed singularities and singularities of polar curves', Math. Ann. 287, 3 (1990), 429-454.

[3] Casas-Alvero (E.). - 'Base points of polar curves', Ann. Inst. Fourier (Grenoble) 41, 1 (1991), 1-10.

[4] Chang (S.H.) and Lu (Y.C.). - 'On $C^{0}$-sufficiency of complex jets', Can. J. Math. 25 (1973), 874-880.

[5] Delgado de la Mata (F.). - 'A factorization theorem for the polar of a curve with two branches', Compositio Math. 92, 3 (1994), 327-375.

[6] García Barroso (E.). - 'Sur les courbes polaires d'une courbe plane réduite', Proc. London Math. Soc. (3) 81 (2000), no. 1, 1-28.

[7] Greuel (G.M.). - 'Constant Milnor number implies constant multiplicity for quasihomogeneous singularities', Manuscripta Math. 56 (1986), no. 2, 159-166.

[8] Henry (J.P.) ANd Merle (M.). - 'Sections planes, limites d'espaces tangents et transversalité de variétés polaires', C.R. Acad. Sc. Paris t. 291 Serie A, (1980), 291294.

[9] Hermann (M.), Ikeda (S.) And Orbanz (U.). - Equimultiplicity and Blowing up, Springer-Verlag, Berlin-Heidelberg-New York, 1988.

[10] Kaup (L.) And Kaup (B.). - Holomorphic Functions of Variables, Gruyter Studies in Math. 3, De Gruyter, Berlin, 1983

[11] LÊ (D.T.). - 'Une application d'un théorème d'A'Campo à l'équisingularité', Indag. Math., 35, 5 (1973), 403-409.

[12] Lê (D.T.), MiChel (F.) AND Weber (C.). - 'Sur le comportement des polaires associées aux germes de courbes planes', Compositio Math., 72 (1989), 87-113.

[13] LÊ (D.T.) AND TEISSIER (B.). - 'Sur la géométrie des surfaces complexes I. tangentes exceptionnelles', Amer. J. Math. 101 (1979), 420-452.

[14] LoOIJENGA (E.J.N.). - Isolated singular points on complete intersections. London Math. Soc. Lecture Note Series, 77. Camb. Univ. Press, Cambridge-New York, 1984.

[15] Luengo (I.). - 'The $\mu$-stratum is not smooth', Invent. Math. 90 (1987), 139-152.

[16] Luengo (I.) ANd Melle Hernández (A.). - 'Une formule pour le nombre de Milnor', C.R. Acad. Sc. Paris t. 321, Série I (1995), 1473-1478. 
[17] Melle Hernández (A.). - 'Milnor numbers for surface singularities', Israel J. Math. 115 (2000) 29-50.

[18] O`ShEA (D.). - 'Topologically trivial deformations of isolated quasihomogeneous hypersurface singularities are equimultiple',Proc. Amer. Math. Soc. 101 (1987), no. 2, 260-262.

[19] Merle (M.). - 'Invariants polaires des courbes planes', Invent. Math. 41 (1977), 103-111.

[20] Teissier (B.). - 'Cycles Evanescents, Sections Planes et Conditions de Whitney'. Singularités a Cargase 1972,Asterisque 7-8, (1973), 363-391

[21] Teissier (B.). - 'Variétés polaires I: invariants polaires des singularités d'hypersurfaces', Invent. Math. 40 (1977), 267-292.

[22] Teissier (B.). - 'Polyèdre de Newton jacobien et équisingularité', Seminar on Singularities (Paris, 1976/1977), Publ. Math. Univ. Paris VII, 7, Univ. Paris VII, Paris, (1980) 193-221.

[23] Teissier (B.). - 'A bouquet of bouquets for a birthday', Topological methods in modern mathematics (Stony Brook, NY, 1991), Publish or Perish, Houston, TX, (1993) 93-122. 